\title{
Broad-spectrum azoles and flucloxacillin: a dangerous match
}

\author{
Tine Vangheluwe $^{1} \cdot$ Frederik Van Hoecke $^{2} \cdot$ Alexander Dumoulin $^{3} \cdot$ Dirk Vogelaers $^{4}$
}

Received: 21 May 2021 / Accepted: 4 August 2021 / Published online: 7 September 2021

(c) The Author(s), under exclusive licence to Springer-Verlag GmbH Germany, part of Springer Nature 2021

\begin{abstract}
Both invasive fungal infection with Aspergillus fumigatus and blood stream infection with methicillin-susceptible Staphylococcus aureus (MSSA) have a significant incidence in the critically ill. Voriconazole and, more recently, isavuconazole and high dose flucloxacillin are the standard first line treatments for these respective serious infections. However, an underestimated risk of a significant interaction needs to be taken into consideration, when both co-occur. We wish to highlight this important issue in the management of these patients through two case reports and to point to the inconsistency between different validated databases regarding this significant interaction as well the importance of a strict protocol for readily available therapeutic drug monitoring.
\end{abstract}

Keywords Voriconazole $\cdot$ Isavuconazole $\cdot$ Flucloxacillin $\cdot$ Interaction

\section{Case reports}

\section{Case 1: interaction between voriconazole and flucloxacillin}

A 72-year-old Caucasian female with chronic lymphatic leukemia and moderate obesity $\left(\mathrm{BMI}=33 \mathrm{~kg} / \mathrm{m}^{2}\right)$ was admitted to the hospital because of SARS-CoV-2 infection. On day 3 of her hospital stay, the patient was transferred to the intensive care unit because of severe Covid-19-related pneumonia and ARDS progressing to respiratory failure sequentially requiring non-invasive CPAP (day 3-4) and invasive BIPAP and prone ventilation with a low $P / f$ ratio $<100$, followed by difficult weaning only achieved on day 43 . Broad-spectrum antimicrobial coverage with ceftriaxone was initiated on day 6 for 9 days because of suspicion of hospital-acquired pneumonia as well as fluconazole on day 9 after isolation of

Tine Vangheluwe

tine.vangheluwe@azdelta.be

1 Departments of Clinical Pharmacy AZ Delta, Deltalaan 1, B-8800 Roeselare, Belgium

2 Department of Laboratory Medicine, Sint Andries Hospital, Bruggestraat 84, B-8700 Tielt, Belgium

3 Department of Intensive Care AZ Delta, Deltalaan 1, B-8800 Roeselare, Belgium

4 Department of Infectious Diseases AZ Delta, Deltalaan 1, B-8800 Roeselare, Belgium
Candida albicans in urine and BAL samples. On day 15, a second BAL yielded growth of Aspergillus fumigatus (BAL 15.66), leading to a diagnosis of probable Covid associated invasive pulmonary aspergillosis (CAPA) and substitution of fluconazole by voriconazole in a classic loading and maintenance dose $(6 \mathrm{mg} / \mathrm{kg}$ bid and $4 \mathrm{mg} / \mathrm{kg}$ bid respectively). On day 18 , high dose flucloxacillin $6 \times 1 \mathrm{~g}$ was initiated because of positive blood cultures for MSSA. The dose of flucloxacillin was increased to $6 \times 2 \mathrm{~g} 2$ days after the therapy was initiated. A first voriconazole level was taken on day 12 of voriconazole therapy, indicating a subtherapeutic level of $<0.2 \mu \mathrm{g} / \mathrm{ml}$, however only available 6 days later. Voriconazole dosing was doubled to $8 \mathrm{mg} / \mathrm{kg}$ BID on day 18 of voriconazole therapy, whereas 4 days earlier flucloxacillin therapy was discontinued after 11 days because of a positive clinical and biochemical evolution. On day 34 , high dose amoxicillin/clavulanic acid was initiated for ten days. A second subtherapeutic voriconazole level of $<0.2 \mu \mathrm{g} / \mathrm{ml}$ was reported 2 days after flucloxacillin interruption, subsequently rising to $3 \mu \mathrm{g} / \mathrm{ml} 3$ days later under the adapted voriconazole dosing regimen.

\section{Case 2: interaction between isavuconazole and flucloxacillin}

A 57-year-old man, with a history of alcohol abuse, diabetes mellitus, and Charcot arthropathy requiring multiple interventions with osteosynthesis, was admitted to hospital 
because of septic shock, originating from diabetic foot, with documented blood stream infection caused by methicillin susceptible Staphylococcus aureus. On day 1, high dose flucloxacillin $(6 \times 2 \mathrm{~g})$ was initiated. Linezolid was associated on day 27. Because of a positive bronchoalveolar lavage galactomannan test (1.5 OD), positive culture from respiratory samples for Aspergillus fumigatus and nodular pulmonary lesions on chest CT scan, as voriconazole was initiated on day 26 , followed by an early switch the day afterwards, because of the prolonged QT interval and decreased renal function. The voriconazole therapy was switched to isavuconazole with a loading dose on the first 2 days. After 7 days of isavuconazole therapy, the serum level proved subtherapeutic $(0.3 \mu \mathrm{g} / \mathrm{mL})$, despite adequate standard dosing. On day 41 linezolid and flucloxacillin were stopped and switched to another broad-spectrum antibiotic because of clinical deterioration without a clear focus of infection.

Isavuconazole was stopped on day 42 because of nontherapeutic level and lack of effect on respiratory function.

On day 77 of the hospitalization, isavuconazole was restarted because of a sharply increased galactomannan value in bronchoalveolar lavage fluid sample (BAL 17.5) and again positive cultures from respiratory samples for Aspergillus fumigatus. As prescribed, the first 2 days of therapy, a loading dose was administered. The isavuconazole serum level, on day 8 of therapy was $1.7 \mu \mathrm{g} / \mathrm{mL}$, indicating a slightly subtherapeutic level. Subsequently, the dosing interval was shortened to $18 \mathrm{~h}$ instead of $24 \mathrm{~h}$ on day 21 of the isavuconazole therapy, when the result of the level became available. On day 25 of the isavuconazole therapy, the level was therapeutic $(5.2 \mu \mathrm{g} / \mathrm{mL})$.

\section{Discussion}

A major proposed mechanism for this significant interaction resides in the activation by flucloxacillin of the pregnane $\mathrm{X}$ receptor (PXR), which in turn induces increased expression of CYP3A4 and CYP2C8/9, responsible for the metabolization of voriconazole [1,2]. Other pathways are being explored for this interaction [3].

The need for therapeutic drug monitoring (TDM) of voriconazole is well established because of non-linear pharmacokinetics, narrow therapeutic-toxic margin, interindividual variability of the expression of the cytochrome enzymes responsible for metabolization and potential for interactions as well as the clinical experience of sub-therapeutic levels in the intensive care unit. We strongly recommend for TDM of voriconazole as described in Patterson TF, et al [4]. Within the institution, this clinical case contributed to a rethinking of the strategy of voriconazole TDM in the intensive care unit, mainly by reassuring the provision of regular thrice-weekly TDM with rapid reporting in order to ensure prompter adaptations. A similar consideration may apply to isavuconazole, especially if combined with other drugs with a potential of interaction.

Clinical pharmacists play a significant role in the multidisciplinary management of serious infections and to this purpose rely on a number of validated databases, including Medicines Complete (linking to Stockley), Up To Date, and Phil [5-7]. At the time of the clinical case, these databases did not mention this significant interaction at all. Furthermore, this interaction is not mentioned in the SmPC neither for voriconazole nor for flucloxacillin. However, there are a number of literature reports warning for this adverse event $[1,2]$.

For the individual patient management one cannot rely sufficiently on a single data base. At the meta-level, we would like to call for an algorithm or peer review system that guarantees a systematic cross check of the different databases and the literature concerning interactions. This would ensure more regular updating and interchangeability of the major databases used in clinical pharmacy for interaction screening. Similar considerations may be needed for isavuconazole, in spite of the initial claim for lesser need of therapeutic drug monitoring using this broad-spectrum azole with linear pharmacokinetics.

\section{Declarations}

Conflict of interest The authors declare no conflict of interest.

\section{References}

1. Kennedy B, Larcombe R et al (2015) Interaction between voriconazole and flucloxacilline during treatment of disseminated Scedosporium apiospermum infection. J Antimicrob Chemother 70(2):2171-2172

2. Bijwerkingen centrum lareb (2019) Interaction voriconazole and flucloxacillin, leading to decreased voriconazole blood levels

3. Muilwijk E, Dekkers B et al (2017) Flucloxacillin results in suboptimal plasma voriconazole concentrations. Antimicro Agents Chemother 6:915-917

4. Patterson TF et al (2016) Practice Guidelines for the Diagnosis and Management of Aspergillosis: 2016 Update by the Infectious Diseases Society of America. Clin Infect Dis 63(4):e1-e60

5. Uptodate interaction checker. Consulted via https://www.uptod ate.com/drug-interactions/?source=responsive_home\#di-drugl ist. Accessed 21 Dec 2020

6. Stockley interaction checker. Consulted via https://www.medic inescomplete.com/\#/interactions/stockley. Accessed 21 Dec 2020

7. Phil interaction checker. Consulted via https://phil.apb.be/nl-BE/ interactions. Accessed 21 Dec 2020

Publisher's note Springer Nature remains neutral with regard to jurisdictional claims in published maps and institutional affiliations. 